\title{
A utilização do teste Morisky-Green na adesão ao tratamento anti- hipertensivo: Deteç̧ão precoce na atenção primária à saúde
}

\author{
The use of the Morisky-Green test in adherence to anti-hypertensive \\ treatment: early detection in primary health care
}

\author{
Marinete Mendes Rosa ${ }^{1}$, Giovanna Areia Leão Nauman ${ }^{2}$, Rosângela Nunes Almeida ${ }^{3}$, Vitor \\ Emanuel Sousa da Silva ${ }^{4}$, Alison de Sousa Moreira ${ }^{5}$, Maria Lindalva Alves da Silva ${ }^{6}$, Valcirlene \\ Mendes Guimarães? 7 , João Victor Almeida Vilanova ${ }^{8}$, Lawanda Kelly Matias de Macedo ${ }^{9}$
}

${ }^{1}$ Acadêmica de Enfermagem. Universidade Estadual do Maranhão (UEMA). Caxias-MA, Brasil. E-mail: marinetymendes@hotmail.com ${ }^{2}$ Acadêmica de Enfermagem. Universidade Estadual do Maranhão (UEMA). Caxias-MA, Brasil. E-mail: gd2627853@gmail.com ${ }^{3}$ Enfermeira. Doutoranda em Enfermagem (UFPI). Mestre em Biodiversidade, Ambiente e Saúde (UEMA). Universidade Estadual do Maranhão (UEMA). Caxias-MA, Brasil. E-mail: rnadasilva@hotmail.com *Autor para correspondência

${ }^{4}$ Acadêmico de Enfermagem. Universidade Estadual do Maranhão (UEMA). Caxias-MA, Brasil. E-mail: gaarakasekaque@gmail.com

${ }^{5}$ Enfermeiro, Especialista em Urgência e Emergência, Coordenador do Serviço de Atendimento Móvel de Urgência. São João do Sóter- MA Brasil.E-mail: alisonsousa2012@bol.com.br

${ }^{6}$ Bióloga. Mestre em Biodiversidade, Ambiente e Saúde. Universidade Estadual do Maranhão(UEMA). Caxias-MA, Brasil. E-mail: lindalva.maria@hotmail.com

${ }^{7}$ Enfermeira. Atua na Força Estadual de Saúde do Maranhão (FESMA), em Marajá do Sena- MA. Especialista em Saúde da Família. E-mail: valmendes06@hotmail.com

${ }^{8}$ Acadêmico de Direito. Centro Universitário de Ciências e Tecnologias do Maranhão (UniFacema). Caxias-MA, Brasil. E-mail: victor1998.vv@gmail.com

${ }^{9}$ Acadêmica de Enfermagem. Universidade Estadual do Maranhão (UEMA). Caxias-MA, Brasil. E-mail: lawandak360@gmail.com

\author{
Palavras-chave \\ Utilização \\ Teste \\ Adesão ao tratamento \\ Anti-hipertensivo \\ Atenção Primária à Saúde
}

A Hipertensão Arterial Sistêmica (HAS) é o principal fator de risco para problemas cerebrovasculares e doenças isquêmicas do coração. Trata-se de um estudo descritivo, intervencionista. $O$ cenário desta investigação foi o município de Aldeias Altas-MA. Participaram da pesquisa 205 portadores de Hipertensão Arterial Sistêmica, tendo como critério de inclusão, estar cadastrado e ser acompanhado pelas Equipes de Saúde da Família. Inicialmente, realizou-se uma reunião com os profissionais de enfermagem atuantes na Atenção Primária à Saúde para apresentação do projeto. Os resultados revelaram que, dos 205 hipertensos partícipes da pesquisa, encontravam-se na faixa etária variando entre 35 a 96 anos, sendo a maioria (66\%) do sexo masculino. Quanto à escolaridade, os maiores percentuais são de pessoas que não sabem lê nem escrever (62\%) e que convivem com companheira (o) e filhos (46\%), $40 \%$ estavam com o índice de massa corporal normal. Na classificação da Pressão Arterial Sistêmica, obteve-se um elevado número (37\%) de pacientes que se encontram com a hipertensão em Estágio 2. Quanto ao tratamento (57\%) faz apenas o uso exclusivo da medicação, (71\%) usam somente uns tipos de drogas, variando de uma a quatro doses diárias, e tendo um tempo de tratamento variando de 1 a 8 anos ou mais. (54\%) não faz uso de tratamento alternativo e os que fazem (46\%) apenas uma pequena parcela $(5 \%)$ deixa de tomar a medicação. Grande parte dos participantes não faz atividade física (75\%) e nem sabem o seu peso ideal (89\%), (56\%) são ex- fumantes, (92\%) não enjerem bebida alcoólica, tendo a comida com pouco sal (70\%), e (99\%) não faz uso de sal em comida ou salada pronta (95\%) não faz uso de saleira em cima da mesa durante as refeições. Quanto ao teste de Morisky-Green, 48 (28\%) foram considerados aderentes, e 157 (77\%) não aderentes ao tratamento medicamentoso. Por se tratar de uma doença que acomete um grande público no município, fica evidente a necessidade do processo educativo junto a esses pacientes e da constante atenção para o esclarecimento da condição de saúde e necessidade de tratamento.

Systemic Arterial Hypertension (SAH) is the main risk factor for cerebrovascular problems and ischemic heart disease. This is a descriptive, interventionist study. The scenario of this investigation was the municipality of Aldeias Altas-MA. 205 patients with Systemic Arterial Hypertension participated in the research, having as inclusion criteria, being registered and being accompanied by the Family Health Teams. Initially, a meeting was held with the nursing professionals working in Primary Health Care to present the project. The results revealed that, of the 205 hypertensive participants in the research, they were in the age group varying between 35 and 96 years, the majority (66\%) being male. As for education, the highest percentages are of people who cannot read or write (62\%) and who live with a partner (o) and children (46\%), $40 \%$ had a normal body mass index. In the classification of Systemic Blood Pressure, a high number (37\%) of patients with Stage 2 hypertension was obtained. As for the treatment (57\%) only uses the medication, (71\%) use only a types of drugs, ranging from one to four daily doses, and having a treatment time ranging from 1 to 8 years or more. (54\%) do not use 
alternative treatment and those who do (46\%) only a small portion (5\%) stop taking the medication. Most of the participants do not do physical activity (75\%) and do not even know their ideal weight (89\%), (56\%) are exsmokers, (92\%) do not drink alcohol, having food with little salt (70\%), and (99\%) do not use salt in food or ready-made salad (95\%) do not use salt on the table during meals. As for the Morisky-Green test, 48 (28\%) were considered adherent, and 157 (77\%) were non-adherent to drug treatment. As it is a disease that affects a large public in the municipality, it is evident the need for an educational process with these patients and constant attention to clarifying the health condition and the need for treatment.

\section{INTRODUÇÃO}

No Brasil, o Ministério da Saúde (MS) destaca que a prevalência estimada de Hipertensão Arterial Sistêmica (HAS) é de $35 \%$ da população acima de 40 anos, o que representa, em números absolutos, um total de 17 milhões de portadores da doença. Entre os idosos, a prevalência é de $50 \%$ da população e cerca de $75 \%$ das pessoas recorrem ao Sistema Único de Saúde (SUS) para receber atendimento, principalmente na Atenção Básica (BRASIL, 2006a).

A HAS é o principal fator de risco para problemas cerebrovasculares e doenças isquêmicas do coração, exigindo tratamento continuo e controle durante toda a vida. No Brasil, em 2003, 27,4\% dos óbitos foram decorrentes de doenças cardiovasculares, atingindo $37 \%$ quando são excluídos os óbitos por causas mal definidas e a violência. Estudo brasileiro revelou que, em indivíduos adultos, 50,8\% sabiam ser hipertensos, $40,5 \%$ estavam em tratamento e apenas $10,4 \%$ tinham pressão arterial controlada, ou seja, valores menores que 140/90 mmHg. Além do mais, a Idade avançada, obesidade e baixo nível educacional mostraram-se associados a essas menores taxas de controle (ALMEIDA et al., 2007).

Com o apoio de uma equipe multiprofissional e também do paciente e de sua família, com diferentes abordagens e a formação de uma equipe interdisciplinar pode proporcionar ação diferenciada, ampliando o sucesso do controle da hipertensão e dos demais fatores de risco cardiovascular, e oferecendo ao paciente e a comunidade uma visão mais ampla do problema. O cuidado envolve também ensinamentos para o conhecimento das doenças, de suas inter-relações, complicações e implica, na maioria das vezes, a necessidade de mudanças nos hábitos de vida e adesão real ao tratamento proposto (CLÁUDIA; LILIANE, 2010).

O controle metabólico rigoroso associado a medidas preventivas e curativas relativamente simples são capazes de prevenir ou retardar o aparecimento das complicações crônicas da hipertensão, resultando em melhor qualidade de vida ao indivíduo. Da mesma forma, o controle pressórico da HAS resulta na redução de danos aos órgãos-alvo, por isso, o manejo dessa enfermidade deve ser feito a partir de um sistema hierarquizado de saúde, em que a sua base precisa ser o nível primário de atendimento (SBH, 1998; PAIVA; BERSUSA; ESCUDER, 2006).

Neste contexto, a Atenção Primária à Saúde (APS), um conjunto de ações voltadas para a prevenção de agravos, promoção de saúde, tratamento e reabilitação, constituindose uma forma de reorganização de serviços que favorece a construção da saúde por meio de uma troca solidária e possível de fortalecer a participação comunitária, o desenvolvimento de habilidades pessoais e a criação de ambientes saudáveis, tornando-se um excelente campo de cuidado aos hipertensos e/ou diabéticos (ANDRADE; BUENO; BEZERRA, 2006).

Ressalta-se que a adesão ao tratamento é considerada um processo complexo, influenciado por fatores ambientais, indivi-duais, de acolhimento por parte dos profissionais de saúde, no qual pela própria doença, encontram-se comprometidas as dimen־sões biológica, sociológica e psicológica. Além destes, somam-se os fatores socioe-conômicos, culturais e comportamentais, dificultando ainda mais o processo de adesão (PIRES; MUSSI, 2008; HELENA; NEMES; ELUF-NETO, 2009).

A não adesão ao tratamento de doenças crônicas é um problema de etiologia multifatorial. Os problemas da adesão verificam-se em todas as situações em que existe autoadministração do tratamento, independentemente do tipo de doença, qualidade e/ou acessibilidade aos recursos da saúde. A crença que os doentes são os únicos responsáveis pela adesão representa um equívoco, dado existirem diversos fatores. Dentre eles destacam-se fatores emocionais o conhecimento do paciente sobre a doença e o seu comportamento frente à tomada dos remédios, que afetam o seu comportamento e a capacidade de adesão ao tratamento (WHO, 2003; BUGALHO; CARNEIRO, 2004).

Portanto, o interesse em realizar este projeto, partiu de vivências em estágio extracurricular na Atenção Primária à Saúde, onde se puderam constatar as dificuldades de adesão do usuário, sobretudo o idoso, ao tratamento da HAS. Além disso, conforme Almeida et al., (2007), verificou-se na literatura a grande repercussão da não-adesão à terapêutica nesse grupo etário, tanto no controle de sintomas, quanto na manutenção da capacidade funcional, levando a importantes 
implicações em sua saúde e em sua qualidade de vida através dessas observações teve como objetivo avaliar a adesão de hipertensos acompanhados na Atenção Primária à Saúde ao tratamento anti-hipertensivo, através da aplicação do Teste de Morisky-Green.

\section{MATERIAL E MÉTODOS}

Trata-se de uma pesquisa avaliativa, com abordagem quantitativa. $O$ estudo avaliativo relaciona-se com a utilização de métodos científicos, com intuito de analisar a relevância da atividade executada, além de seus efeitos, os rendimentos que foram produzidos para auxiliar na tomada de decisões, e também deve considerar as perspectivas dos diferentes atores envolvidos para analisar adequação estratégica entre a intervenção e o problema que a originou (CONTANDRIOPOULOS et al., 1997). Portanto, avaliar consiste em medir, comparar e possuir fundamentos para emitir juízo de valor.

A abordagem quantitativa foi utilizada por possibilitar gerar medidas precisas e confiáveis que permitirão uma análise estatística do fenômeno estudado; neste caso as informações coletadas com os pacientes hipertensos, acompanhados pela APS (LUNA, 2002).

O cenário deste estudo foi o município de Aldeias AltasMA, com população estimada em 2017 de 26387 habitantes. Apresenta uma área de unidade territorial de $1951 \mathrm{~km}^{2}$ e faz parte da região Leste do Maranhão. Localiza-se há $370 \mathrm{Km}$ da Capital, São Luís, e, há 125 Km de Teresina, capital do Piauí (IBGE, 2017).

Em relação a Atenção Primária em Saúde, o município conta com 09 Equipes de Saúde da Família, distribuídos em: 03 UBS localizadas na Zona Urbana e 05 na Zona Rural (CNESnet, 2018).

Participaram do projeto, portadores de hipertensão arterial, acompanhadas pela Atenção Primária à Saúde, no município de Aldeias Altas-MA. O critério de seleção foi ser acompanhado pela estratégia, residir na zona urbana, está apto mentalmente para responder o instrumento de pesquisa

Para a coleta de dados, utilizar-se-á um formulário adaptado e o Teste de Morisky-Green, contendo questões estruturadas e semiestruturadas, acerca da doença e tratamento medicamentoso e não medicamentoso. Este é um método de avaliação da adesão à terapia medicamentosa, com relato do próprio usuário, sendo de simples aplicação e de fácil entendimento, fornecendo indícios sobre as barreiras da adesão (BARBOSA, 2007).

O Teste Morisky- Green, permite mensurar o grau de adesão do tratamento. Trata-se de uma escala composta por quatro perguntas que abordam os seguintes itens: se alguma vez o paciente esquece de tomar o remédio, se é descuidado com o horário das tomadas dos medicamentos, se quando está se sentindo bem deixa de tomar o medicamento e se quando se sente mal deixa de tomá-lo. O grau de adesão é determinado por pontuação das respostas: $\operatorname{Sim}=0$ e Não $=1$ e os resultados variam de 0 a 4, correspondendo, respectivamente, o menor valor aos não aderentes e o maior valor aos aderentes. Assim, o critério adotado para pontuação será de 0 a 3 pontos para os menos aderentes e 4 pontos para os mais aderentes (MORISKY; GREEN; LEVINE, 1986; STRELEC; PIERIN; MION, 2003).

Para a coleta dos dados utilizou-se de um formulário, contendo questões gerais sobre os dados Sócio-demográficos e antropométricos de hipertensos cadastrados na APS, Investigação da presença de comorbidades e fatores que prejudicam o tratamento da Hipertensão Arterial Sistêmica, aplicação de medidas intervencionistas, como atividades lúdicas, na adesão ao tratamento da HAS, e também o teste de Morisky- Green. Portanto, a coleta dos dados ocorreu entre os meses de outubro 2018 a janeiro de 2019, nas 03 unidades de saúde da zona urbana, com os pacientes hipertensos acompanhados na APS.

Os pacientes hipertensos, foram abordados pelos pesquisadores participante na sala de espera das UBS, e após terem sido atendidos, foram convidados a participar da pesquisa.

Os dados obtidos com a aplicação dos formulários, foram agrupados por categorias, por ordem de importância, buscando-se a maior organização das informações e melhor visualização dos achados.

Quanto aos aspectos éticos legais os sujeitos participantes, receberam previamente informações a fim de esclarecer a justificativa, objetivos e finalidades, enfatizando a relevância da pesquisa, e, somente foi iniciada a aplicação do formulário após a assinatura do Termo de Consentimento Livre e Esclarecido (TCLE), no qual assegura todo o sigilo acerca das informações fornecidas.

\section{RESULTADOS E DISCUSSÃO}

De início ocorreu uma reunião com os profissionais de enfermagem atuantes na Atenção Primária à Saúde, especificamente nas Unidades Básicas de Saúde Centro de Saúde (com 02 equipes de Saúde da Família), UBS Vila Costa Pinto e UBS São Francisco, para apresentação do projeto.

Participaram da pesquisa 205 hipertensos que se disponibilizaram a responder o formulário, a abordagem desses hipertensos ocorreu na sala de espera das seguintes Unidades Básicas de Saúde, Centro de saúde, São Francisco e 
Vila Costa Pinto na qual foi explicado a metodologia e os objetivos da pesquisa e da disponibilidade dos mesmos em responder ao formulário sendo sim é feita a coleta das informações.

Ao termino da aplicação do teste Morisky- Green era feita a leitura e entrega de um folder contendo informações sobre a HAS para os participantes.

As características sociodemográficas do público estudado estão apresentadas nas respectivas tabelas abaixo:

A idade dos 205 pacientes incluídos na pesquisa variou em média de 35 a 96 anos, a maioria pertencente ao sexo feminino (66\%). Este dado também foi observado em outro

Tabela 1. Distribuição dos 205 hipertensos participantes segundo variáveis socioeconômicas. Aldeias Altas - MA, 2019.

\begin{tabular}{lll}
\hline VARIÁVEIS & n & \% \\
\hline Idade & & 36 \\
Até 60 anos & 73 & 64 \\
Mais de 60 anos & 132 & \\
Gênero & & 34 \\
Masculino & 69 & 66 \\
Feminino & 136 & \\
Naturalidade & & 84 \\
Maranhão & 173 & 13 \\
Piauí & 27 & 1 \\
Sergipe & 1 & 2 \\
Ceará & 4 & \\
Residência & & 100 \\
Zona Urbana & 205 & 0 \\
Zona Rural & 0 &
\end{tabular}

\section{Escolaridade}

Não alfabetizado $\quad 127 \quad 62$

$\begin{array}{lll}\text { Alfabetizado } & 30 & 14\end{array}$

Ensino Fundamental Incompleto $\quad 33 \quad 16$

Ensino Fundamental Completo $\quad 4 \quad 2$

Ensino Médio Incompleto $\quad 6 \quad 3$

Ensino Médio Completo $\quad 4 \quad 2$

Ensino Superior Incompleto $\quad 1 \quad 1$

Situação Conjugal

Convive com companheira (o) e filhos $\quad 95 \quad 46$

Convive com companheira (o) com laços conjugais sem

filhos

$6-3$

Convive com companheira (o), filhos e/ou outros familiares

$6-3$

Convive com familiares sem companheira (o) 80

Vive só

$80 \quad 39$

Ocupação

Comerciante

Aposentado

Empregada (o) doméstica (o)

Do lar

1

Outra

$148 \quad 72$

TOTAL

$1 \quad 1$

$15 \quad 7$

$40 \quad 19$

Fonte: Dados do autor, 2019 
estudo de idosos com HA como (DINIZ, 2009).

Para Zaitune et al (2006) a maior ocorrência de HAS em mulheres dessa faixa etária se deve, geralmente, à maior percepção das doenças e à tendência ao autocuidado. Destaca-se, também, a maior procura do serviço de saúde pelas mulheres em comparação aos homens, assim aumenta a probabilidade de se ter a HAS diagnosticada.

Quanto à escolaridade os maiores percentuais são de pessoas que não sabem lê nem escrever (62\%). O analfabetismo está presente como fator dificultador da adesão ao tratamento medicamentoso (Matos, 2009; MEDEIROS et al., 2011; MARQUES et al., 2010), pois, por mais que pacientes tenham interesse em modificar hábitos de vida e desejem seguir a prescrição e orientação da equipe de saúde, há momentos em que isso se torna impossível, pela incapacidade de leitura e compreensão da prescrição.

Em relação à situação conjugal maior parte dos entrevistados convive com companheira(o) e filhos (46\%) e são aposentados conforme mostra tabela 1. Estudos nesse sentido enfatizam que é relevante o envolvimento dos familiares no tratamento (BELLATO, 2016).

A família representa um importante aliado no tratamento da HAS, sendo percebida por muitos como agente facilitador no processo de adesão ao tratamento e incentivadora na adoção de práticas de autocuidado, por exemplo, na realização de exercícios físicos, no uso de uma alimentação apropriada, além de acompanhá-los em seu itinerário terapêutico (BARRETO; MARCON, 2016).
Quanto ao $\mathrm{O}$ Índice de Massa Corporal dos analisados (40\%) estavam com o IMC normal, (37\%) com sobrepeso, (16\%) com obesidade classe I, (5\%) com obesidade classe II, (1\%) com obesidade classe III (1\%) com baixo peso. A hipertensão arterial constitui fator associado à obesidade, ou seja, indivíduos com hipertensão arterial têm a razão de 3,62 vezes mais chances de serem obesos. (AMER, 2011).

O sobrepeso e a obesidade, associados à baixa escolaridade, constituem os fatores relacionados à hipertensão arterial frequentemente detectado nos estudos brasileiros (GUS, et al.,2004). A hipertensão arterial, por sua vez, é uma das mais importantes causas de morbimortalidade e um predominante fator de risco para o desenvolvimento de doenças cardiovasculares (MINISTÉRIO DA SAÚDE, 2006).

Na classificação da Pressão Arterial Sistêmica, (15\%) dos hipertensos estavam com a PAS normal, (20\%) em estágio de Pré- Hipertensão, (26\%) com hipertensão estágios 1 e (37\%) em estagio 2 , e (2\%) estavam com a pressão abaixo dos parâmetros normais, conforme a tabela 2 .

De acordo com a tabela 3, o tratamento dos portadores de hipertensão arterial sistêmica do estudo, é (42\%) medicamentoso associado, (57\%) faz uso exclusivo medicamentoso e (1\%) não faz uso de medicação, cerca de (71\%) fazem o uso de 1 tipo de droga (25\%) dois tipos de drogas (3\%) usam três tipos de drogas e (1\%) não usa medicação, quanto ao número de doses (37\%) faz uso de apenas uma dose, (43\%) duas doses, (15\%) três doses, (4\%) tomam 4 doses diárias e (1\%) não usam a medicação. Tendo

Tabela 2. variáveis do índice de massa corporal e da classificação da PA dos 205 hipertensos participantes. Aldeias Altas-MA, 2019.

\begin{tabular}{lll}
\hline VARIÁVEIS & n & \% \\
\hline Classificação do índice de massa corporal - IMC & & \\
Baixo peso $(<18,5)$ & 1 & 1 \\
Normal $(18,5-24,9)$ & 83 & 40 \\
Sobrepeso $(\geq 25$ e $\leq 29,9)$ & 76 & 37 \\
Obeso Classe I $(\geq 30$ e $\leq 34,9)$ & 32 & 16 \\
Obeso Classe II $(\geq 35$ e $\leq 39,9)$ & 11 & 5 \\
Obeso Classe III $(\geq 40)$ & 2 & 1 \\
Classificação da pressão & & \\
Pressão baixa & 5 & 2 \\
Normal (PAS $<120$ PAD $<80)$ & 30 & 15 \\
Pré-hipertensão (PAS 120-139 PAD 80-89) & 40 & 20 \\
Hipertensão estágio I (PAS 140-159 PAD 90-99) & 54 & 26 \\
Hipertensão estágio II (PAS $\geq 160-179$ PAD $\geq 100-$ & \\
109) & 76 & 37 \\
\hline TOTAL & $\mathbf{2 0 5}$ & $\mathbf{1 0 0 \%}$ \\
\hline
\end{tabular}

Fonte: Dados do autor, 2019. 
Tabela 3. Variáveis sobre o tratamento dos 205 hipertensos participantes. Aldeias Altas-MA, 2019.

\begin{tabular}{|c|c|c|}
\hline VARIÁVEIS & $\mathbf{n}$ & $\%$ \\
\hline \multicolumn{3}{|l|}{ Tratamento } \\
\hline Não medicamentoso & 1 & 1 \\
\hline Medicamentoso exclusivo & 118 & 57 \\
\hline Medicamentoso associado & 86 & 42 \\
\hline \multicolumn{3}{|l|}{ Número de drogas } \\
\hline 1 droga & 146 & 71 \\
\hline 2 drogas & 52 & 25 \\
\hline 3 drogas & 6 & 3 \\
\hline Não usa & 1 & 1 \\
\hline \multicolumn{3}{|l|}{ Número de doses diárias } \\
\hline 1 dose & 77 & 37 \\
\hline 2 doses & 89 & 43 \\
\hline 3 doses & 30 & 15 \\
\hline 4 doses ou mais & 8 & 4 \\
\hline Não usa & 1 & 1 \\
\hline \multicolumn{3}{|c|}{ Tempo de tratamento (anos) } \\
\hline 1 ano & 28 & 14 \\
\hline 2 anos & 17 & 8 \\
\hline 3 anos & 7 & 3 \\
\hline 4 anos & 16 & 8 \\
\hline 5 anos & 11 & 5 \\
\hline 6 anos & 11 & 5 \\
\hline 7 anos & 2 & 1 \\
\hline 8 anos ou mais & 81 & 40 \\
\hline Não sabe & 32 & 16 \\
\hline \multicolumn{3}{|c|}{$\begin{array}{l}\text { Já parou de fazer o tratamento alguma } \\
\text { vez? }\end{array}$} \\
\hline Sim & 68 & 33 \\
\hline Não & 137 & 67 \\
\hline TOTAL & 205 & $100 \%$ \\
\hline
\end{tabular}

Fonte: Dados do autor, 2019

Tabela 4. Uso de tratamento alternativo nos 205 hipertensos participantes. Aldeias Altas-MA, 2019.

\begin{tabular}{lll}
\hline VARIÁVEIS & $\mathbf{n}$ & $\mathbf{\%}$ \\
\hline Uso de tratamento alternativo & & \\
Sim & 95 & 46 \\
Não & 110 & 54 \\
Quando você usa o tratamento alternativo, você deixa & & \\
de tomar a medicação & 11 & 5 \\
Sim & 84 & 41 \\
Não & $\mathbf{2 0 5}$ & $\mathbf{1 0 0 \%}$ \\
\hline TOTAL &
\end{tabular}

Fonte: Dados do autor, 2019. 
seguindo de um número significante de pacientes que não

sabem há quanto tempo faz tratamento.

Em relação à doença, destaca-se a sua cronicidade e quanto ao tratamento medicamentoso, o fato de o tratamento ser para toda vida, os efeitos indesejáveis das drogas e posologias complexas, no tratamento não

Tabela 5. Tratamento não medicamentoso e hábitos de vida dos 205 hipertensos participantes. Aldeias Altas-MA, 2019.

\begin{tabular}{lll}
\hline VARIÁVEIS & n & \% \\
\hline Atividade física regular três vezes por semana & & \\
Regular & 23 & 11 \\
Irregular & 28 & 14 \\
$\begin{array}{l}\text { Ausente } \\
\text { Pratica-se atividade física qual? }\end{array}$ & 154 & 75 \\
$\begin{array}{l}\text { Caminhada } \\
\text { Ciclismo }\end{array}$ & 42 & 21 \\
Outra & 5 & 2 \\
Você sabe qual seu peso ideal? & 4 & 2 \\
Sim & 22 & \\
Não & 183 & 11 \\
Você fuma? & & 89 \\
Sim & 17 & \\
Não & 74 & 36 \\
Foi fumante & 114 & 56 \\
Se fuma, já tentou parar alguma vez? & & \\
Sim & 12 & 6 \\
Não & 5 & 2 \\
Você tem o costume de tomar bebida alcóolica? & & \\
Sim, todos os dias & 1 & 1 \\
Sim, finais de semana & 14 & 7 \\
Não & 190 & 92 \\
Se sim, durante a última semana você tomou? & & \\
Cerveja/chope & 9 & 5 \\
Vinho \\
Cachaça
\end{tabular}

Você tem alguma dificuldade para reduzir sua ingestão de bebida alcoólica?

Sim

Não

1

14

7

Como é a quantidade de sal em sua comida?

Normal

$57 \quad 28$

Fraca/pouca

$144 \quad 70$

Muita (bem temperada)

42

Você tem o hábito de adicionar sal a comida pronta ou salada?

$\begin{array}{lll}\text { Sim } & 86 & 42 \\ \text { Não } & 119 & 58\end{array}$

Em sua casa a saleira fica em cima da mesa nas refeições?

\begin{tabular}{lll} 
Sim & 11 & 5 \\
Não & 194 & 95 \\
\hline TOTAL & $\mathbf{2 0 5}$ & $\mathbf{1 0 0 \%}$ \\
\hline
\end{tabular}

Fonte: Dados do autor, 2019 
medicamentoso, a necessidade de mudanças em hábitos e estilos de vida também podem ser aspectos que dificultam a adesão (DA SILVA et al., 2016).

A tabela 4, mostra que (54\%) não faz o uso de tratamento alternativo e dos que fazem (46\%) apenas (11\%) deixa de tomar a medicação.

De acordo com os dados colhidos, verificamos um grande índice de hipertensos muitos dos quais fazem uso de plantas medicinais como tratamento. A origem do conhecimento em relação à utilização de plantas medicinais para grande parte da população que faz uso das mesmas como práticas de saúde, normalmente ocorre entre as pessoas mais idosas (LOPES et al., 2010).

Conforme a tabela 5, a maioria dos entrevistados (75\%) não faz atividade física e nem sabem o seu peso ideal (89\%) e (56\%) já fumou, (92\%) não enjerem bebida alcoólica, (70\%) faz uso de pouco sal na comida, quanto ao uso de sal em comida ou salada pronta (58\%) não faz, e (95\%) não tem saleira em cima da mesa durante as refeições.

Cabe ressaltar que as causas mais prováveis da hipertensão arterial são os hábitos alimentares, baixos níveis de exercício físico, ingestão de sal em excesso, obesidade e o consumo álcool exagerado (ORGANIZAÇÃO PAN-AMERICANA DA SAÚDE, 2003).

De acordo com Medina e colaboradores (2010), a pratica de atividades físicas regularmente é fundamental nas intervenções não farmacológicas de prevenção e tratamento da hipertensão arterial. Entre alguns benefícios que a atividade física proporciona a pessoas hipertensas estão a redução da angina; melhora na falta de ar de pessoas com insuficiência cardíaca, melhora na qualidade de vida, ajuda no controle da pressão arterial em pacientes com doenças coronarianas, reduz em um quarto a mortalidade em pessoas que apresentam infarto do miocárdio (BORTH; SARTORI; SIQUEIRA, 2010). Apresentam outras comorbidades tais como osteoporose, gastrite e outras conforme a tabela 6 .

Em relação ao teste de Morisky-Green considera-se não aderente um escore igual a quatro. Dos 205 pacientes, 157 (77\%) não aderiram ao tratamento medicamentoso, pois respondeu "sim" à pela menos uma das quatro perguntas do questionário. Por conseguinte, 48 (23\%) responderam "não" para todas as perguntas do Teste de Morisky-Green, esses foram os pacientes que são aderentes ao tratamento.

Vários fatores externos impedem a adesão, como falta de tempo para tomar os medicamentos ou para consulta médica, custo do tratamento, custo de uma dieta saudável, falta de plano de saúde e esquecimento, como observado no nosso estudo (MARSHALL, 2012).

Um estudo realizado em Ribeirão Preto SP com pacientes idosos com hipertensão, acompanhados pelo serviço público de saúde, revelou que $58 \%$ de idosos hipertensos interromperam o tratamento da
HAS devido aos efeitos adversos dos medicamentos. O tratamento farmacológico deve ser efetivo, ter pouco ou nenhum efeito adverso e não interferir negativamente na qualidade de vida, ao contrário, pode levar ao abandono do tratamento ou fazê-lo de forma irregular (BASTOS et al., 2012).

Ocorreram também algumas ações que teve como objetivo levar mais esclarecimento para esse público sobre a HAS.

O encontro com um grupo de idosos foi passível ter uma discussão sobre a doença e seus agravos e medidas de como preveni-las. Momento de levar orientações esclarecer dúvidas sobre mitos e verdades sobre a HAS e a importância da tomada da medicação conforme a prescrição medica.

Com a ação na praça intitulada "Eu me cuido", ocorrida na Praça Gonçalves Dias, fio dado orientações sobre a HAS, feito aferição de PA, Medidas antropométricas. Com isso foi possível atingir um público que não tem o habito de frequentar UBS, aja vista que aferimos pressão de pessoas que são diagnosticadas com HAS mais que abandonaram o tratamento, e estavam há muito tempo sem verificar a PA.

Com a visita feita com alguns ACS na casa de alguns idosos portadores de hipertensão foi possível levar informações sobre a doença, esclarecimento de dúvidas sobre a sua medicação e posologia a importância de se seguir o tratamento e os riscos do não cumprimento da prescrição médica.

No decorrer dos encontros foi verificado que muito hipertenso tomam mais de um tipo de droga para o tratamento da hipertensão, devido alguns relatos de troca de medicação se pensou em fazer saquinhos personalizados (figura 1) para evitar confusão na hora da tomada das doses diárias da medicação, foram distribuídos com o auxílio dos ACS 300 saquinhos personalizados, dando preferência para aqueles que são idosos e analfabetos, por ser o público com maior tendência em fazer essa troca de medicação.

Figura 1. Saquinhos personalizados entregues aos portadores de hipertensão arterial sistêmica.

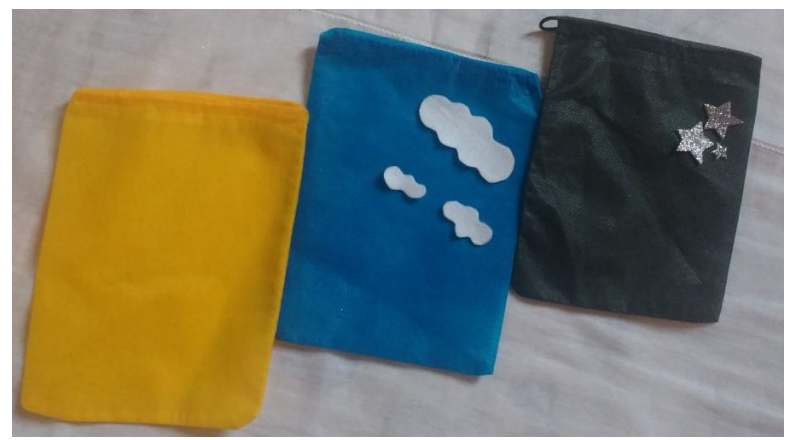

Fonte: Dados do autor, 2019. 


\section{CONCLUSÃO}

Com a aplicação do teste de Morisky- Green ficou evidente que a maioria dos hipertensos não são aderentes a medicação, muitos relataram deixar de tomar a medicação por esta se sentindo bem e por não saber que a hipertensão se trata de uma doença crônica em que o tratamento e continuo. Foi perceptível também que muitos fazem confusão na tomada da medicação principalmente o público idoso e analfabeto.

Por se tratar de uma doença que acomete um grande público no município fica evidente a necessidade permanente do processo educativo junto a esses pacientes e da constante atenção para o esclarecimento da condição de saúde e necessidade de tratamento.

\section{REFERÊNCIAS}

ALMEIDA, V. et al. A hipertensão arterial. Manual de atenção à saúde do adulto - Hipertensão e diabete. Belo Horizonte: Secretaria de Estado de Saúde de Minas Gerais, 2 ed. p. 17-65; 151-62, 2007.

AMER, N.M.; MARCON, S.S.; SANTANA, R.G. Índice de massa corporal e hipertensão arterial em indivíduos adultos no Centro-Oeste do Brasil. Arq Bras Cardiol, v. 96, n. 1, p. 4753, 2011.

ANDRADE, L.O.M.; BUENO, I.C.H.C.; BEZERRA, R.C. Atenção Primária à Saúde e Estratégia Saúde da Família. In: CAMPOS, G. W. S. et al. Tratado de Saúde Coletiva. São Paulo: Hucitec; Rio de Janeiro: Fiocruz, p. 783-836, 2006.

ANTCZAK, S.E. et al. Fisiopatologia Básica. [Trad. Ana Karine Ramos Brum]. Rio de Janeiro: Guanabara Koogan, 2005.

BARBOSA, R.G.B. Estudo da adesão ao tratamento e do controle da pressão arterial em idosos hipertensos. 2007. Dissertação (Mestrado) - Programa de Pós-Graduação da Faculdade de Medicina de Ribeirão Preto, Universidade de São Paulo, Ribeirão Preto, 2007.

BARRETO, S.M.; MARCON, S.S.; Participação familiar no tratamento da hipertensão arterial na perspectiva do doente. Rev Texto \& Contexto Enferm [Internet]. 2014 [cited 2016 Mar 18];23(1):[about 5 p]. Available from: http://www.scielo.br/pdf/tce/v23n1/pt_0104 -0707-tce23-01-00038.pdf.

BASTOS-BARBOSA, R.G. et al. Adesão ao tratamento e controle da pressão arterial em idosos com hipertensão. Arq Bras Cardiol, 2012;99(1):636-41.

BELLATO, R.et al. Experiência familiar de cuidado na situação crônica. Rev Esc Enferm USP [Internet]. 2016 [cited 2016 Mar 16];50(Spe.) :[about 5 p]. Available from: http://www.scielo.br/pdf/reeusp/v50nspe/pt_ _0080- 6234-reeusp-50-esp-0081.pdf

BORTH, J.F.; SARTORI, L.I. M.; SIQUEIRA, P.C.M. Os benefícios da atividade física para hipertensos. EFDeportes - Revista Digital. Ano 15. Núm.147. 2010

BRASIL. Ministério da Saúde. A implantação da Unidade Saúde. In: Cadernos de Atenção Básica: Programa Saúde da Família. Departamento de Ações Básicas/ Secretária de Políticas de Saúde, Brasília: Ministério da Saúde, 2012.

BUGALHO, A.; CARNEIRO, A. V. Intervenções para Aumentar a Adesão Terapêutica em Patologias Crônicas. Lisboa: Centro de Estudos de Medicina Baseada na Evidência, 2004.

CLÁUDIA, C.N.F. A.; LILIANE, M. Acompanhamento farmacoterapêutico a hipertensos e diabéticos na unidade de saúde Tereza Barbosa: análise de caso. Rev Bras Farm Hosp. Serv Saúde São Paulo, São Paulo, v. 1 n. 1, p. 1-24, set/dez, 2010.

DA SILVA, S.S.B. E. et al. O controle da hipertensão arterial em mulheres e homens: uma análise comparativa. Revista da Escola de Enfermagem da USP, v. 50, n. 1, p. 50-58, 2016.

DINIZ M.A.; TAVARES, D.M.S.; RODRIGUES, L.R. Características sócio-demográficas e de saúde entre idosos com hipertensão arterial. Ciênc. cuid. saúde [Internet]. 2009 [cited 2011 jun 30];8(4):607-14.

GUS, I.et al. Prevalência, reconhecimento e controle da hipertensão arterial sistêmica no estado do Rio Grande do Sul. Arq Bras Cardiol. 2004; 83 (5): 424-8.

HELENA, E.T.S.; NEMES, M. I; ELUF-NETO, J. Avaliação da assistência a pessoas com hipertensão arterial em unidades de estratégia saúde da família. Saúde Soc, São Paulo, v. 19, n. 3, p. 614-26, jul/set, 2009.

IBGE. Instituto Brasileiro de Geografia e Estatística. Cidades. [s.l.:s.n.], 2017. Disponível em: < http:// www.ibge.gov.br/cidades >. Acesso em: 02 de maio de 2018.

LOPES, G.A.D. et al. Plantas medicinais: indicação popular de uso no tratamento de hipertensão arterial sistêmica (HAS). Revista Ciência em Extensão, p. 143-155, 2010.

MALTA, D.C. et al. A construção da vigilância e prevenção das doenças crônicas não transmissíveis no contexto do Sistema Único de Saúde. Epidemiol Serv Saúde, v. 15, n. 3, p. 47-65, 2006.

MARSHALL. J.; WOLFE, C.D.A.; MCKEVITT C. Lay perspectives on hy-pertension and drug adherence: systematic review of qualitative research. BMJ. 2012;345:e3953. doi: 10.1136/ bmj.e3953

MARQUES, E.I.W. et al. Motivos da não adesão ao tratamento médico prescrito entre os idosos de uma unidade de saúde da família do município de Passo Fundo-RS. Revista Brasileira de Ciências do Envelhecimento Humano, Passo Fundo, v. 7, n. 2, p. 267-279, maio-ago. 2010. 
MATOS, C.R. Pictogramas e seu uso nas instruções médicas: estudo comparativo entre repertórios para instruções de uso de medicamentos. Dissertação (Mestrado em Ciências da Comunicação) - Escola de Comunicações e Artes da Universidade de São Paulo, São Paulo, 2009.

MEDEIROS, G.C.R. et al. Pictogramas na orientação farmacêutica: um estudo de revisão. Revista Brasileira de Farmácia, Rio de Janeiro, v. 93, n. 2, p. 96-103, 2011.

MEDINA, F.L. et al. Atividade física: impacto sobre a pressão arterial. Revista Brasileira de Hipertensão. Vol. 7. p. 103106. 2010.

MELCHIORS, A.C. Hipertensão Arterial: análise dos fatores relacionados com o controle pressórico e a qualidade de vida. Dissertação (Mestrado em Ciências Farmacêuticas) Universidade Federal do Paraná. Curitiba, PR, 2008.

MINISTÉRIO DA SAÚDE. Secretaria de Atenção à Saúde. Departamento de Atenção Básica. Hipertensão arterial sistêmica para o Sistema Único de Saúde. Brasília; 2006. (Cadernos de Atenção Básica; no 16/ Série A. Normas e Manuais Técnicos).

MORISKY, D.E.; GREEN. L.W.; LEVINE, D.M. Concurrent and predictive of a self-reported measure of medication adherence. Med Care, v. 24, n. 1, p. 67-74, 1986.

ORGANIZAÇÃO PAN-AMERICANA DA SAÚDE (OPAS). Doenças crônico-degenerativas e obesidade: estratégia mundial sobre alimentação saudável, atividade física e saúde. Brasília, DF. 2003.

PAIVA, D.C.P.; BERSUSA, A. A. S.; ESCUDER, M. M. L. Avaliação da assistência ao paciente com diabetes e/ou hipertensão pelo Programa Saúde da Família do Município de Francisco Morato, São Paulo, Brasil. Cadernos de saúde pública, v. 22, p. 377-385, 2006.

PIRES, C.G.S.; MUSSI, F.C. Crenças em saúde para o controle da hipertensão arterial. Ciênc Saúde Colet., Rio de Janeiro, v. 13, n. 2, p. 2257-67, mar/abr, 2008.

SBC - Sociedade Brasileira de Cardiologia; SBH - Sociedade Brasileira de Hipertensão; SBN - Sociedade Brasileira de Nefrologia. V Diretrizes de Hipertensão Arterial. 2006. Disponível em: < http://www.sbn.org.br/Diretrizes/V_Diretrizes_Brasileira s_de_Hipertensao_Arterial.pdf >. Acesso em 01 jul 2012.

SBH, S.B.C. SBN. Sociedade Brasileira de Hipertensão, Sociedade Brasileira de Cardiologia, Sociedade Brasileira de Nefrologia. III Consenso Brasileiro de Hipertensão Arterial. Campos do Jordão (Brasil): SBH, SBC e SBN, 1998. SMELTZER, S.C.; BARE, B. G. Brunner \& Suddarth: Tratado de Enfermagem Médico-cirúrgica. [trad. José Eduardo Ferreira de Figueiredo]. 11. ed. v. 2. Rio de Janeiro: Guanabara Koogan, 2009.

STRELEC, M.A.A. M.; PIERIN, A.M.G.; MION, D. A influência do conhecimento sobre a doença e a atitude frente à tomada dos remédios no controle da Hipertensão Arterial. Arq Bras Cardiol, v. 81 n. 4, p. 343-348, 2003.

UNGARI, A.Q. Adesão ao tratamento farmacológico de pacientes hipertensos seguidos nos núcleos de saúde da família do município de Ribeirão Preto, SP. Universidade de São Paulo, [dissertação] Mestrado em Ciências Médicas. Ribeirão Preto, 2007.

VI Diretrizes Brasileiras de Hipertensão - DBH VI. Rev Bras Hipertens, São Paulo, v.17, n. 1, p. 7-10, jan/mar, 2010.

WILLIAMS, B. The year in hypertension. JACC, v. 55, n. 1, p. 6673, 2010.

WHO - World Health Organization. Adherence to long-term therapies: evidence for action; 2003.

ZAITUNE, M.P.A. et al. Hipertensão arterial em idosos: prevalência, fatores associados e práticas de controle no Município de Campinas, São Paulo, Brasil. Cad Saúde Pública. 2006;22(2):285-94.

Submissão: 03/04/2020

Aprovado para publicação: 18/04/2020 